\title{
RETRACTED ARTICLE: Adaptation strategies of farmers to drought in a changing climate in Dingcheng County, Hunan, southern China
}

\author{
Yongdeng Lei $\cdot$ Hongjian Zhou $\cdot$ Jing'ai Wang • \\ Weixia Yin · Bin Tang $\cdot$ Huimin Yang
}

Received: 2 July 2013/Accepted: 3 November 2013/Published online: 19 November 2013

(c) Springer-Verlag Berlin Heidelberg 2014

The peer-review process for the above article was found to have been compromised and inappropriately influenced by the corresponding author, Yongdeng Lei. As a result the findings and conclusions of these articles cannot be relied upon.

The Editor-in-Chief was misled in believing that he had accepted the article based upon a favorable review. The article is retracted based upon violation of the integrity of the peer review process.

The corresponding author and the publisher wish to retract this paper to preserve the integrity of material published in the journal. The publisher acknowledges that the integrity of the peer review process should have been subject to more rigorous verification to ensure the reviews provided were genuine and impartial. The publisher apologizes for any inconvenience rendered to the readers of the journal and wishes to assure the reader that measures have been taken to ensure that the peer review process is comprehensively checked to avoid a similar error occurring.

Y. Lei · J. Wang $(\bowtie)$

State Key Laboratory of Earth Surface Processes and Resource

Ecology, Beijing Normal University, Beijing 100875, China

e-mail: jwang@bnu.edu.cn

Y. Lei

e-mail: leiyongdeng568@gmail.com

Y. Lei $\cdot$ J. Wang $\cdot$ W. Yin $\cdot$ B. Tang $\cdot$ H. Yang

School of Geography, Beijing Normal University,

Beijing 100875, China

e-mail: yinwx@mail.bnu.edu.cn

B. Tang

e-mail: 734864092@qq.com

H. Yang

e-mail: hmyang09@163.com

Y. Lei · J. Wang $\cdot$ W. Yin $\cdot$ H. Yang

Key Laboratory of Regional Geography, Beijing Normal

University, Beijing 100875, China

H. Zhou

National Disaster Reduction Center of China (NDRCC),

Beijing 100022, China

e-mail: zhouhongjian@ndrcc.gov.cn 